\title{
Free-induction-decay magnetometer based on a microfabricated Cs vapor cell
}

\author{
D. Hunter ${ }^{1}$, S. Piccolomo ${ }^{1}$, J. D. Pritchard ${ }^{1}$, N. L. Brockie ${ }^{2}$, T. E. Dyer ${ }^{2}$ and E. Riis ${ }^{1}$ \\ ${ }^{1}$ Dept. of Physics, University of Strathclyde, Glasgow G4 ONG, UK ${ }^{*}$ \\ ${ }^{2}$ Texas Instruments, Larkfield Industrial Estate, Greenock PA16 OEQ, UK
}

(Dated: May 23, 2018)

\begin{abstract}
We describe an optically pumped Cs magnetometer containing a $1.5 \mathrm{~mm}$ thick microfabricated vapor cell with nitrogen buffer gas operating in a free-induction-decay (FID) configuration. This allows us to monitor the free Larmor precession of the spin coherent Cs atoms by separating the pump and probe phases in the time domain. A single light pulse can sufficiently polarize the atomic sample however, synchronous modulation of the light field actively drives the precession and maximizes the induced spin coherence. Both amplitude and frequency modulation have been implemented with noise floors of $3 \mathrm{pT} / \sqrt{\mathrm{Hz}}$ and $16 \mathrm{pT} / \sqrt{\mathrm{Hz}}$ respectively within the Nyquist limited bandwidth of $500 \mathrm{~Hz}$.
\end{abstract}

\section{INTRODUCTION}

Optically pumped magnetometers monitor the precession frequency of polarized spins in an atomic vapor to infer the magnitude of an external magnetic field. Advancements in optical pumping techniques [1, 2] enable extremely precise measurements through observation of variations in the absorption or polarization rotation of transmitted light 3. These highly sensitive devices have significant potential in numerous applications including: geophysical surveying, biomedical imaging such as magnetocardiography (MCG) and magnetoencephalography (MEG), and in fundamental research [4 6]. Silicon wafer technology provides a reliable, consistent and high-throughput method of constructing Cs cells that can be easily integrated into a packaged device, in comparison to traditional glass-blown cells [7. The miniaturized nature of microfabricated magnetometers introduces numerous advantages such as reduced power consumption, portability and low cost of fabrication whilst maintaining a higher level of performance than existing technologies of similar size and power requirements [8]. Although many glass-blown cells can be operated at room temperature, effectively removing the need for heating which is required in miniaturized cells to achieve an appreciable absorption, they are limited in terms of manufacturability and also scalability resulting in poor spatial resolution if applied to potential sensor networks 9]. Also, effective heat loss management can dramatically reduce the power required to heat these micofabricated devices to as low as $11 \mathrm{~mW}$ [10].

The most sensitive atomic sensors, such as the spinexchange relaxation free (SERF) magnetometer, typically operate in a shielded environment and have demonstrated aT $/ \sqrt{\mathrm{Hz}}$ sensitivities [1]. Although sensitive, these vector sensors operate near zero field at high atomic densities, limiting the dynamic range and elevating the power required to heat the vapor cell. In contrast, total field sensors including the FID, Bell-Bloom and $\mathrm{M}_{\mathrm{x}}$ implementations have a relatively large dynamic range enabling operation in Earth-field conditions with typical

\footnotetext{
* dominic.hunter@strath.ac.uk
}

sensitivities at the $\mathrm{pT} / \sqrt{\mathrm{Hz}}$ level depending on the vapor cell dimensions [8, 12.

Sensitivity of atomic magnetometers is proportional to the magnetic linewidth arising from numerous sources of spin relaxation both inherent to the vapor cell and induced by external perturbations. Fundamentally, SERF sensors are limited by spin-destruction collisions hence the extensive use of $\mathrm{Rb}$ and $\mathrm{K}$ with their smaller collisional cross-sections in comparison to Cs 13 . The resulting narrow magnetometer linewidth will dramatically improve the sensitivity at the expense of a considerably limited bandwidth (typically $<100 \mathrm{~Hz}$ ) compared to other microfabricated atomic sensors with relaxation rates in the $\mathrm{kHz}$ range. Most atomic magnetometry systems are limited by spin-exchange collisions therefore Cs was the choice of atomic species in this work as its higher vapor pressure results in lower power requirements.

Optically pumped magnetometer systems that utilize lock-in detection typically require an initial estimation of the field before sweeping across the magnetic resonance. This can be quite difficult in an unshielded environment where the ambient fields are unpredictable and prone to fluctuations. The FID mechanism is desirable as it can be implemented in a single-pulse regime in which the magnetic field information is readily extracted from the observed transient oscillation without any prior knowledge of the field. An alternative approach is to utilize synchronous optical pumping whereby the light-field is modulated at the Larmor frequency as adopted by Bell and Bloom [2. A sensitivity of $1 \mathrm{pT} / \sqrt{\mathrm{Hz}}$ has been shown with an all-optical Bell-Bloom magnetometer using independent pump and probe beams in a sensing volume of $16 \mathrm{~mm}^{3} 12$.

Many atomic magnetometry schemes operate in a cw regime were the spin preparation (pump) and detection (probe) stages are performed simultaneously with a single laser beam [14, 15]. Here we discuss a pulsed approach implemented in either the amplitude modulation (AM) or frequency modulation (FM) configurations. Separating these distinct phases in the time domain allows the polarized spins to precess freely at the Larmor frequency whilst decaying exponentially as a consequence of various spin decoherence phenomena. The spin dynamics associated with the precession and relaxation can also 
be viewed as an oscillation and decoherence of atomic population among the ground-state Zeeman sublevels. It should be noted that the AM and FM techniques are special cases of a perturbation implemented by optical pumping, and that other sources of modulation can be applied. As this technique monitors the precession directly, it provides significant advantages in accuracy over driven magnetometers that are subject to systematic frequency shifts imposed by phase errors in the feedback signal 16. It also obviates the need for demodulation and tracking of the modulation frequency. It has been demonstrated that the FM technique provides similar performance to the $\mathrm{M}_{\mathrm{x}}$-magnetometer with the added advantage of being fully optical 8 . This is particularly beneficial in applications that require multiple adjacent sensors as cross-talk is not an issue.

In this paper we present the application of the FID technique using the AM and FM implementations with a microfabricated Cs vapor cell. The concept of differential polarimetry detection in a FID setting is discussed along with the efficiency of optical pumping in the single-pulse and synchronous regimes. Finally, the relative sensitivity performance in a static field of similar magnitude to that of the Earth's is measured for both configurations. This analysis is performed by determining the Cramer-Rao lower bound (CRLB) using a single FID trace [17 19], and also through calculation of the root spectral density (RSD) acquired from subsequent fits of FID data in a signal train.

\section{VAPOR CELL FABRICATION AND TESTING}

The sensor head for atomic magnetometers typically comprises of a cell filled with magnetically sensitive alkali atoms in the vapor form [7]. The fabrication process for the cells used in this work involves etching a $1.5 \mathrm{~mm}$ thick silicon wafer, compatible with large-scale production, to form the cell cavities. The holes are created using a wet etch process producing a cavity with a trapezoidal cross-section; the sides are etched in the $\{111\}$ crystal direction wheras the bottom resides in the $\{100\}$ plane resulting in an angle of $35.3^{\circ}$ with respect to the vertical direction. Glass is bonded to one side and the cells are filled with a caesium azide $\left(\mathrm{CsN}_{3}\right)$ water-based solution [20]. After evaporation, a second stage of anodic bonding seals the top face of the wafer with an upper glass surface. An example of the final result is shown in Fig. 1 (a). Following a method suggested in the literature [21, 22], the entire wafer is exposed to UV light in order to dissociate the azide into its constituents; nitrogen as a buffer gas and Cs in the metallic form. As the UV process progresses, the Cs vapor starts to saturate the cell and we observe droplets of metallic Cs condensing on the glass walls of the cells. Fig. 11 (b) shows the presence of Cs droplets on the upper glass surface of a single cell. Crystallized azide residual is visible close to the borders of the lower glass surface.

We use absorption spectroscopy to deduce the amount of $\mathrm{N}_{2}$ content within the microfabricated Cs vapor cell
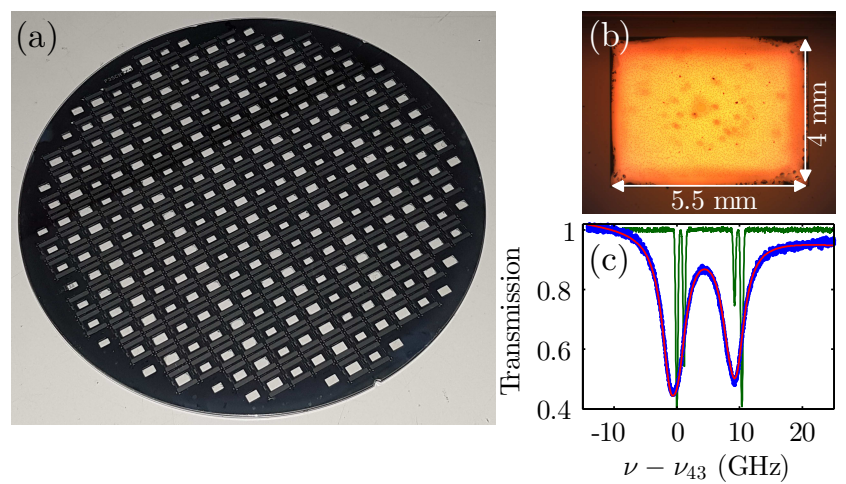

FIG. 1. (a) An example of a $1.5 \mathrm{~mm}$ thick silicon wafer, $8^{\prime \prime}$ in diameter, containing numerous undiced cells. (b) Cavity of a typical vapor cell; condensed Cs vapor is clearly visible with azide residual mostly accumulating around the sides of the vapor cell. (c) Spectroscopic signal (blue) and corresponding fit (red) for the vapor cell implemented in the magnetometer systems reported here. The frequency axis is scaled relative to the $F=4 \rightarrow F^{\prime}=3$ transition shown in the reference signal (green).

and obtain precise information on the azide dissociation process. The spectroscopic properties are determined by directing a linearly polarized light beam through the vapor cell which is heated to $85^{\circ} \mathrm{C}$. The laser intensity is kept below the resonant saturation intensity $\mathrm{I}_{\mathrm{sat}} \simeq 25 \mu \mathrm{W} / \mathrm{mm}^{2}$ to avoid power broadening. A reference setup was used to normalise the intensity variation of the laser and to provide a relative and absolute frequency reference using an etalon and auxiliary Cs cell. The transmission spectrum is modelled using the BeerLambert law which introduces the total absorption crosssection given by 23 ,

$$
\sigma(\nu)=\frac{9 \lambda_{0}^{2}}{4 \pi} \frac{\Gamma_{0}}{2(2 I+1)} \sum_{F, F^{\prime}} C_{F F^{\prime}}^{2} f_{v}\left(\Delta_{F F^{\prime}}, \Gamma_{g}, \Gamma_{l}\right)
$$

where $I=7 / 2$ is the nuclear spin of Cs, $\lambda_{0} \simeq 894.59 \mathrm{~nm}$ and $\Gamma_{0} \simeq 2 \pi \cdot 4.56 \mathrm{MHz}$ are the wavelength and natural linewidth of the $D_{1}$ line respectively 24, $C_{F F^{\prime}}$ are the relative line strengths for transitions from $F \rightarrow F^{\prime}$, and $f_{v}\left(\Delta_{F F^{\prime}}, \Gamma_{g}, \Gamma_{l}\right)$ is the normalised lineshape associated with each hyperfine transition.

In the presence of $\mathrm{N}_{2}$ buffer gas the optical resonance is modified by two phenomena proportional to the pressure: homogenous broadening resulting in a Lorentzian lineshape $\Gamma_{l} \rightarrow \Gamma_{0}+\Gamma_{N_{2}}$, and a characteristic shift of the transition frequencies represented by the modified detuning $\Delta_{F F^{\prime}} \rightarrow \nu-\nu_{F F^{\prime}}+\Delta_{N_{2}}$ 25]27. The slightly temperature dependent ratio between these two parameters can be viewed as a characteristic of the particular buffer gas which for $\mathrm{N}_{2}$ is approximately $\Gamma_{N_{2}} / \Delta_{N_{2}} \simeq-2.3$ at $85^{\circ} \mathrm{C}$. The Voigt lineshapes are modelled in accordance with Ref. 28] by calculating the convolution of the Lorentzian $\Gamma_{l}$ and Gaussian $\Gamma_{g}$ profiles arising from the collisional and Doppler broadening mechanisms respectively. The fit model is applied to the absorption data using the 
(a)

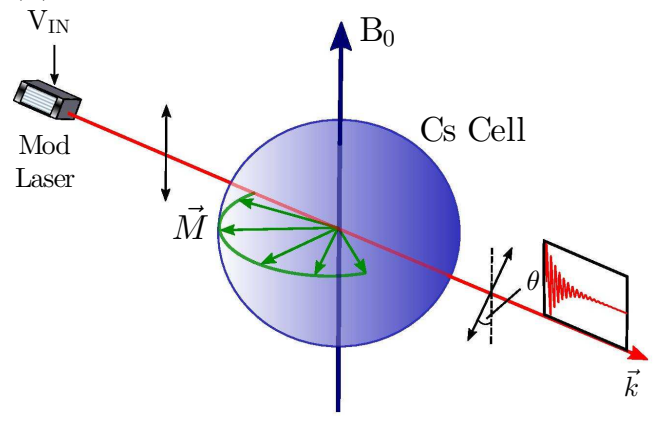

(b)

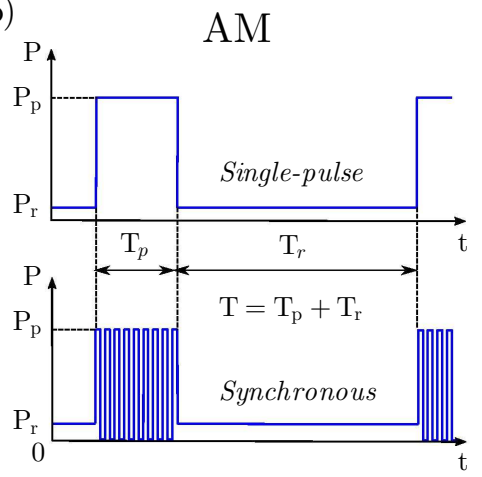

(c)

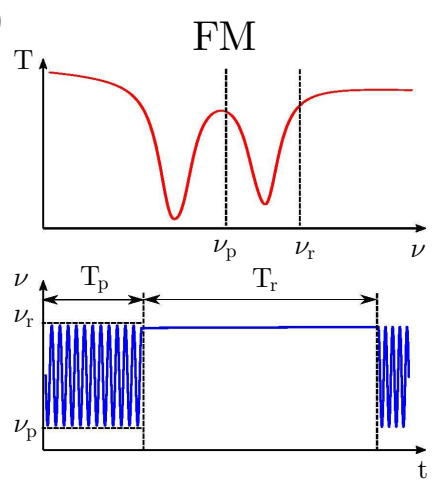

FIG. 2. (a) Experimental model conveying the basic mechanisms behind a FID magnetometer. The laser is modulated to alter the absorption coefficient with a strong atom-light interaction building up spin polarization for a time $T_{p}$ during the pump phase. The interaction strength is then lowered for a time $\mathrm{T}_{\mathrm{r}}$ and the time-dependent optical rotation angle is monitored. (b) AM configuration showing the switching of laser power from high to low. Two possible modulation inputs (single-pulse or synchronous) can be applied to the modulator with a single pump-probe cycle occurring at the driving frequency $1 / \mathrm{T}=\mathrm{f}_{\mathrm{d}}$. (c) Spectroscopic signal illustrating the pump $\nu_{p}$ and probe $\nu_{r}$ frequencies used in the FM implementation. Laser frequency is sinusoidally modulated over the $F=3 \rightarrow F^{\prime}$ transition in the synchronous regime. A single pulse can also be applied by setting $\nu_{p}$ to the centre of the resonance peak.

Levenberg-Marquardt algorithm to extract the relevant fit parameters, primarily the broadening and shift with respect to the standard Cs spectrum in the absence of buffer gas. Fig. 1 (c) shows an example of an absorption signal recorded from the sensor head with the corresponding numerical fit; it can be seen that collisional broadening has merged the transitions resulting in a two-peak spectrum with a slight overlap. The $\mathrm{N}_{2}$ pressure can be estimated based on the broadening which was calculated to be $\Gamma_{N_{2}} \simeq 2 \pi \cdot 2.74 \mathrm{GHz}$. Neglecting the temperature dependence of the collisional broadening rate, the buffer gas pressure was estimated to be 165 Torr using a conversion of $16.64 \mathrm{MHz} /$ Torr [26].

Ultimately, magnetometer sensitivity is limited by the size of the vapor cell and the fabrication process can be optimized by adjusting the $\mathrm{N}_{2}$ pressure based on the cavity dimensions. For example, the amount of azide content can be varied as well as the UV light dose providing numerous potential broadening values ranging from a resolvable four peak spectrum to a fully broadened single resonance. One peak cells, where all four hyperfine resonances have been merged together, are typically bonded in a $\mathrm{N}_{2}$ atmosphere and can exceed optical linewidths of $\Gamma_{N_{2}}>20 \mathrm{GHz}$. The etching process can also be modified to produce different cavity volumes with the wall relaxation rate highly dependent on the smallest cavity dimension. Based on the cell size, optical linewidth and experimental parameters the spin relaxation rate for the vapor cell implemented here was calculated to be $\gamma_{20} \simeq 1.5 \mathrm{kHz}$ in the absence of power broadening; spin-exchange collisions were the dominant relaxation contribution at the optimal operating temperature in terms of magnetometer sensitivity [29].

\section{FID MAGNETOMETRY}

FID is a well understood phenomenon with the first optically detected signal in an alkali vapor achieved by Dehmelt 30, who demonstrated that the precession of sodium atoms generated by an external magnetic field would induce a modulation in the absorption of a circularly polarized probe. This was adequately described using the Bloch formalism with the evolving macroscopic magnetization altering the absorption coefficient of the atomic vapor. The FID mechanism is also conveniently utilized in numerous NMR experiments that manipulate nuclear, rather than electronic, spins. Analogies in the model, observed data and signal processing techniques can be drawn from this highly recognized field.

Fig. 2 conveys the physical principles behind the FID magnetometer in both the AM and FM configurations. Optical pumping with circularly polarized light creates a population imbalance in the Zeeman levels of the hyperfine ground state which classically is analogous to the creation of a net magnetization $\boldsymbol{M}$ along the direction of the laser beam. An external magnetic field will induce Zeeman splitting of the hyperfine ground states resulting in precession of the magnetization at the Larmor frequency $\omega_{L}=\gamma\left|\boldsymbol{B}_{\mathbf{0}}\right|$, where $\gamma \simeq 2 \pi \cdot 3.5 \mathrm{~Hz} / \mathrm{nT}$ is the gyromagnetic ratio of the Cs atomic ground state. The magnetometer bandwidth is limited to half the driving frequency $f_{d}$ in accordance with the Nyquist theorem. Optical pumping is performed at a peak pump power $\mathrm{P}_{\mathrm{p}}$ for a time $T_{p}$ enabling the build-up of spin coherence in the atomic ensemble. This process can be made more efficient by employing synchronous modulation [2] where the light intensity is modulated at the Larmor frequency or one of its subharmonics. The light level is then reduced to a readout power $\mathrm{P}_{\mathrm{r}}$ for a time $\mathrm{T}_{\mathrm{r}}$ to adequately moni- 
tor the precession whilst reducing perturbations through residual optical pumping. For the FM implementation the laser frequency is sinusoidally modulated at the Larmor frequency during the pump phase, extending over the full $F=3 \rightarrow F^{\prime}$ transition as shown in Fig. 2 (c). The centre frequency of the sinusoidal waveform was very close to the absorption peak therefore one might expect the strongest resonance to occur at a modulation frequency of $\omega_{L} / 2$. This is not the case as the broadened optical linewidth causes simultaneous excitation of all the hyperfine transitions with the optimized modulation residing at $\omega_{L}$ for the pump and probe frequencies shown here. The probe is blue detuned to the side of the $F=3 \rightarrow F^{\prime}$ resonance producing a weak atom-light coupling that is analogous to the low-intensity stage considered in the AM configuration. All other parameters including pump duration, input polarization and beam width were kept consistent with that of the AM scheme to make a valid comparison of the performance of both techniques.

The Bloch equations provide an intuitive model for the evolution of the macroscopic magnetization oscillating in the plane transverse to the external magnetic field,

$$
\dot{M}=\gamma \boldsymbol{M} \times \boldsymbol{B}_{0}-\gamma_{2} \boldsymbol{M}
$$

where $\gamma_{2}$ is the total polarization decay rate comprising numerous contributions including depolarizing collisions with the cell walls, spin-exchange and residual optical pumping effects [16]. This is of course a vectorial model and does not take into account tensor (alignment) contributions generated by the high-intensity circularly polarized light beam however, the resulting time-dependent solutions are useful as they relate closely to experimental observations. A full treatment regarding the evolution of atomic polarization can be modelled using the density matrix formalism describing the dynamics of the atomic ground state [31]. The optical rotation angle is proportional to the degree of spin polarization along the beam propagation direction 32] with the solution,

$$
M_{x}(t)=M_{0} \sin \left(\omega_{L} t+\phi_{0}\right) e^{-\gamma_{2} t},
$$

which resembles a decaying sinusoid with a frequency corresponding to the precession experienced by the Cs atoms.

\section{AMPLITUDE MODULATION SCHEME}

A schematic of the experimental arrangement is shown in Fig. 3 which can be extended to both the AM and FM configurations. The sensor head consists of a microfabricated vapor cell with a trapezoidal cavity, the dimensions of which are shown in Fig. 1 (b). The vapor cell temperature is controlled by supplying a gated current to a resistive heating element, with measurements performed during the off state to avoid spurious magnetic field contributions. Increasing the cell temperature will improve the signal-to-noise ratio (SNR) as the atomic density rises. However, this will also reduce the spin

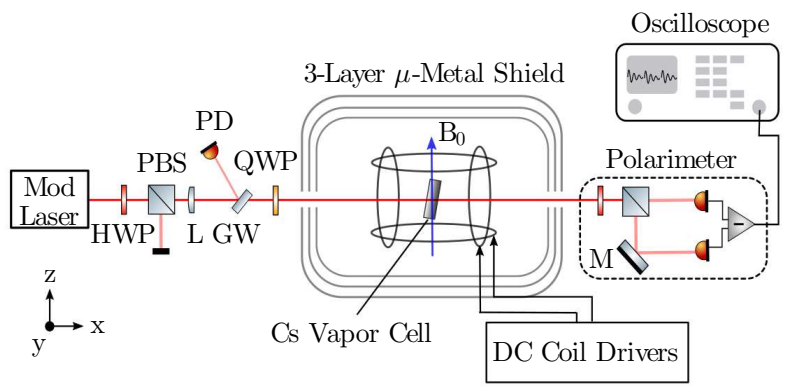

FIG. 3. Experimental set-up for a FID magnetometer with a microfabricated $\mathrm{Cs}$ vapor cell containing $\mathrm{N}_{2}$ buffer gas. AM is achieved using an AOM whereas FM is performed by modulating the injection current of a VCSEL. The direction and magnitude of $\mathrm{B}_{0}$ is controlled by supplying separate DC currents to each Helmholtz coil. (VCSEL: vertical cavity surface emitting laser, PBS: polarising beam splitter, HWP: halfwave plate, QWP: quarter-wave plate, AOM: acousto-optic modulator, GW: glass window, M: mirror, L: lens, PD: photodiode.

coherence time due to the higher number of collisions. The counterplay of these two parameters results in an optimum sensitivity occuring at $\approx 85^{\circ} \mathrm{C}$ for the thermal vapor cells considered here. The sensor head is placed inside a 3-layer $\mu$-metal shield, nulling the ambient magnetic field to several nT and highly suppressing technical noise contributions. A set of two-axis Helmholtz coils driven by a stable DC current supply (powered by a $12 \mathrm{~V}$ car battery) were designed to generate a uniform bias field, surpassing that of the Earth's $(\sim 50 \mu \mathrm{T})$, pointing along a user-defined direction in the $\mathrm{x}-\mathrm{z}$ plane.

An extended cavity diode laser (Toptica DL pro) is manually tuned to the $D_{1}$ line using an auxiliary Cs reference cell. The fibre-coupled output is passed through a half-wave plate and PBS to ensure maximum transmission of linearly polarized light through the transmitted channel. The quarter-wave plate then introduces an adjustable circular component before illuminating the vapor cell. Laser power is varied using the first order deflection of an AOM output driven by a frequency mixer; the control voltage input is provided by a programmable arbitrary function generator (Keysight 33500B series) and the RF input is supplied by a signal generator (Marconi 2022). The diameter of the beam entering the AOM is carefully managed to optimize the dynamic range with the lowest optical power on the order of $1 \mu \mathrm{W}$. The pump and probe powers are monitored using reflection from a glass window with a biased photodiode. The probe power inside the vapor cell was kept at $\mathrm{P}_{\mathrm{r}} \approx 200 \mu \mathrm{W}$ with a measured beam diameter of $1.8 \mathrm{~mm}$ for both the AM and FM implementations. The best sensitivity was achieved at the highest peak pump power $\mathrm{P}_{\mathrm{p}} \approx 6 \mathrm{~mW}$ available from the laser suggesting that this is slightly below the optimum value in terms of system performance. The laser frequency was tuned to the centre of the merged $F=3 \rightarrow F^{\prime}$ absorption line of the broadened two-peak hyperfine resonance as it was discovered that larger probe 

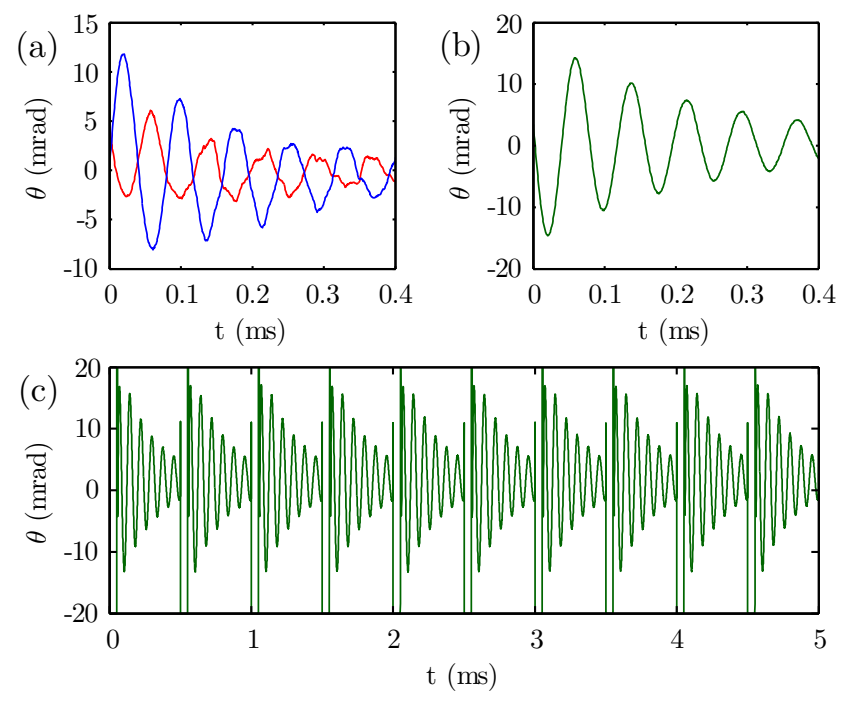

FIG. 4. FID signals (raw data) extracted from the polarimeter with (a) the transmitted (blue) and reflected (red) channels of the PBS, and (b) the difference signal (green) showing the optical rotation experienced in a bias field of $3.7 \mu \mathrm{T}$. Intensity noise is significantly reduced due to common-mode noise rejection. (c) A snapshot of a typical FID train for the final polarimetry signal. In this case, the driving frequency was set to $2 \mathrm{kHz}$ leading to signal truncation.

intensities could be implemented before power broadening occurred, in comparison to the $F=4 \rightarrow F^{\prime}$ transition, resulting in an improved magnetometer sensitivity. An increase in spin coherence was also observed which is a consequence of operating in the light-narrowed regime causing repumping of atoms out of the $F=3$ ground state, whilst simultaneously probing the $F=4$ hyperfine manifold with a weak atom-light interaction as a consequence of being adequately frequency detuned from resonance 33 .

A balanced polarimeter was utilized as a dispersive measurement detecting optical rotation in the transmitted light, induced by the component of the macroscopic magnetization $M_{x}(t)$ precessing transverse to the magnetic field. This modifies the birefringent properties of the sample causing the two orthogonal circular contributions of the linearly polarized component of the light beam to experience different refractive indices. This differential measurement is only possible due to the phase opposition of the two signals shown in Fig. 4 (a) which are the projections of the $y$ and $z$ components of the output light. Optical rotation in the $\mathrm{y}-\mathrm{z}$ plane will cause a variation in the projection of one of these components at the expense of the other, resulting in the $\pi$ phase difference observed between the two signals. It can be noticed that the amplitudes of both components are not exactly equal; this is attributable to the elliptical polarization of the input light coupled with the time-dependent anisotropy of the sample.

A typical polarimeter consists of a half-wave plate, PBS (separating the linearly polarized y and $\mathrm{z}$ components), and two photodiodes as shown in Fig. 3. Appli-

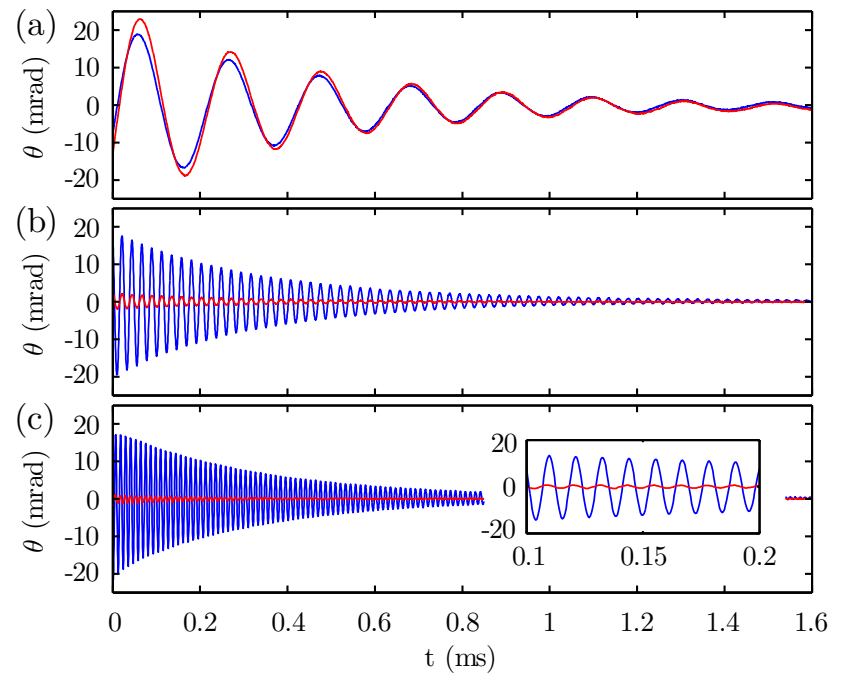

FIG. 5. Optical rotation signal (raw data) observed using single-pulse (red) and synchronous (blue) optical pumping for bias fields of (a) $1.5 \mu \mathrm{T}$, (b) $10 \mu \mathrm{T}$ and (c) $25 \mu \mathrm{T}$. The inset in plot (c) shows $100 \mu$ s of data to adequately resolve the oscillations resulting from the precessing atomic spins. The total measurement period was set to $T=2 \mathrm{~ms}$ showing the full decay of the atomic spin polarization to equilibrium.

cation of a half-wave plate in the detection process allows an equal proportion of the light through both channels of the PBS. Low light levels are to be expected during the probe phase therefore both photodiode signals are amplified before subtraction to form the final polarimetry signal. Since a single beam is employed for both the pumping and probing processes, elliptically polarized light provides an adequate balance for this detection mode; the circular component is used to prepare the atomic spins and the linear component enables detection of the evolving magnetization through optical rotation. The degree of ellipticity of the input light will affect the efficiency of the optical pumping and detection processes and it was found that approximately an equal superposition of circular and linear polarization was optimal 34. The noise floor of the polarimeter was measured to be $0.1 \mu \mathrm{rad} / \sqrt{\mathrm{Hz}}$, with a $300 \mathrm{kHz}$ detection bandwidth, for the probe intensity considered here. If adequately balanced, a polarimeter is usually more sensitive than absorption-based detection as the differential measurement results in suppression of common-mode noise, including laser intensity fluctuations. This is clearly evident by comparing Figs. 4 (a) and (b) which convey a large degree of noise cancellation when the individual outputs of the PBS are subtracted, as well as an increase in signal amplitude. It is also important to note that the sum of these two signals would result in an absorption measurement as is the case with using a single photodiode. A section of the final polarimetry signal is shown in Fig. 4 (c) in the form of a FID signal train allowing tracking of the magnetic field in the time domain.

The FID traces displayed in Fig. 5 were generated in different bias fields using both single-pulse and syn- 
chronous optical pumping. The data was recorded at a sampling rate of $f_{s}=2 \mathrm{MHz}$ using a Tektronix DPO5034 digital phosphor oscilloscope with 12 bit voltage resolution. The arbitrary waveform generator used to drive the AOM enabled both pumping techniques to be implemented in consecutive pump-probe cycles. This ensured consistency in the system parameters being applied and allowed a detailed investigation of the two optical pumping techniques. Square wave modulation provided a larger SNR in the AM implementation which is most likely a consequence of the increased time spent at peak pump power. Modulation methods tend to require prior knowledge of the external field however, this can be easily attained in an FID setting by scanning the modulation frequency and maximizing the signal amplitude in the time domain. Alternatively, single-pulse optical pumping can be utilized to initially estimate the Larmor frequency, that can then be inserted as the modulation input in a two-step feedback process. Single-pulse optical pumping was found to be more efficient at low magnetic fields where the Larmor frequency was on the order of the relaxation rate. This is simply a consequence of the pump time $T_{p}$ restricting the number of oscillations available in the synchronous regime at low frequencies. Optical pumping using a single pulse becomes increasingly inefficent at stronger bias fields which prompts a reduction in signal amplitude. These observations are consistent with the analytical solutions provided in Ref. [16]. Transverse magnetic fields depolarize the atomic spins therefore synchronously driving the precession is essential in maintaining a consistent degree of phase coherence at large bias fields. This can be readily seen in Fig. 5 where the signal amplitude remains constant at higher Larmor frequencies. The magnetometer linewidth was found to be $\gamma_{2} \simeq 3 \mathrm{kHz}$ suggesting that power broadening has occurred as a consequence of the elevated probe intensities.

Fig. 6 (a) illustrates the build up of spin polarization, proportional to the FID amplitude, as the number of pulses in the synchronous regime is increased in the AM configuration. The data was fit to the model, $\mathrm{A}_{\theta}=\mathrm{a}_{\theta}\left(1-e^{-\gamma_{\mathrm{op}} \mathrm{T}_{\mathrm{p}}}\right)$, resulting in an optical pumping rate of $\gamma_{\mathrm{op}} \simeq 8.5 \mathrm{kHz}[16$. The total spin polarization was estimated to be $\mathrm{P} \approx 0.74$ using a simple four-level model that takes into account the rapid collisional mixing of atoms in the excited state arising due to the presence of $\mathrm{N}_{2}$ [13. A more accurate model would account for all 32 Zeeman sublevels of the $\mathrm{D}_{1}$ line which would be described by the evolution of a $32 \times 32$ density matrix [35]. Saturation of the spin polarization is clearly evident at $\mathrm{A}_{\theta} \simeq 27 \mathrm{mrad}$ resulting in an optimum noise density of $\rho_{\mathrm{B}_{0}} \simeq 2 \mathrm{pT} / \sqrt{\mathrm{Hz}}$. It can be readily seen that there is little improvement in sensitivity as the pump duration surpasses a single relaxation period as the sample has become sufficiently polarized.

\section{SENSITIVITY ANALYSIS}

Fig. 7 (a) shows a single FID trace in a magnetic field of $50 \mu \mathrm{T}$, transverse to the beam propagation direction, that has been post-processed by fitting the data to the
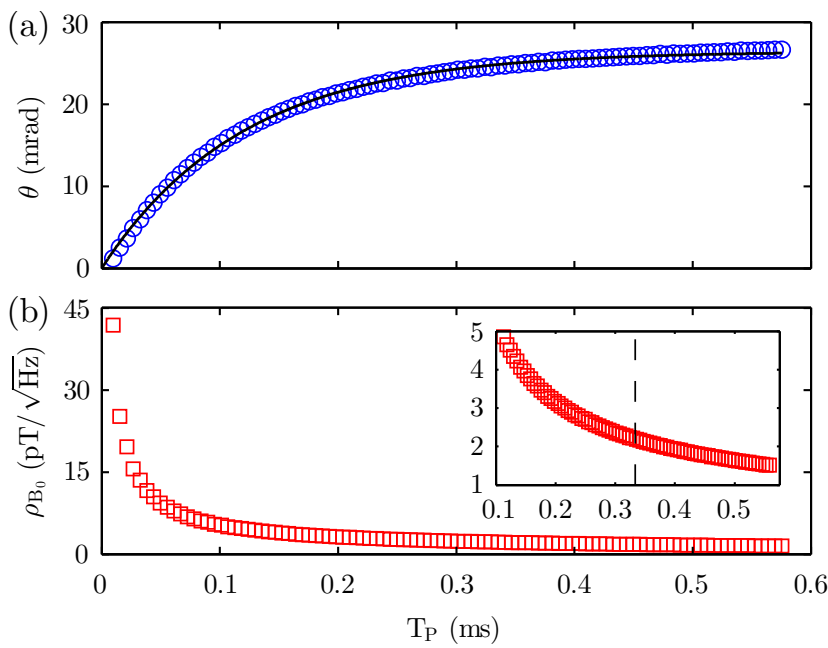

FIG. 6. Dependence of the (a) FID amplitude (blue) and (b) CRLB estimated noise density (red) as a function of pump duration in the synchronous regime. The black line in (a) is the associated fit to an exponential model. The number of cycles during the pump stage was swept from 1 to 100 for a fixed driving frequency. The dashed line in (b) indicates the pump duration used in the sensitivity analysis and is close to a single relaxation period.

model given in Eq. (3). The relevant experimental parameters were extracted including: signal amplitude $\mathrm{A}_{\theta}$ (proportional to the initial magnetization $\mathrm{M}_{0}$ ), angular Larmor frequency $\omega_{L}$, damping rate $\gamma_{2}$ and initial phase $\phi_{0}$. The signal noise $\sigma_{\theta} \simeq 50 \mu \mathrm{rad}$, used in the CRLB estimations, was calculated from the polarimeter response at the same probe power as previous experiments. The FID signal train was recorded over a period of $1 \mathrm{~s}$ with the fitting routine applied to each individual FID trace providing time domain magnetic field data as shown in Fig. 7 (b). Any DC offsets in the raw signals are a consequence of imperfect balancing of the polarimeter and this can be added to the fit model as an extra parameter. Alternatively, since we are only interested in the part of the signal oscillating at $\omega_{L}$, a bandpass Butterworth filter can be applied in the software to smooth the previously recorded data as well as eradicate any DC offsets or slowly varying terms. A discrete time Fourier transform (DTFT) has also been attempted to extract the relevant frequency content however, the optimum linewidth achievable in the microfabricated cells provide a fundamental limit on the frequency resolution. Applying the DTFT to a full signal train results in numerous peaks with a spacing $f_{d}$, and the strongest peak occurring at a multiple of $f_{d}$ closest to the Larmor frequency. Fitting the data to a damped sinusoid model is therefore the most robust and high resolution method of inferring the precession frequency for these vapor cells.

The Cramer-Rao lower bound (CRLB), given by the standard deviation $\sigma_{B_{0}}$, is a measure of the statistical uncertainty of determining the field from a single FID trace [18. It is possible to reduce this noise by averaging over successive magnetic field measurements extracted from 
(a)
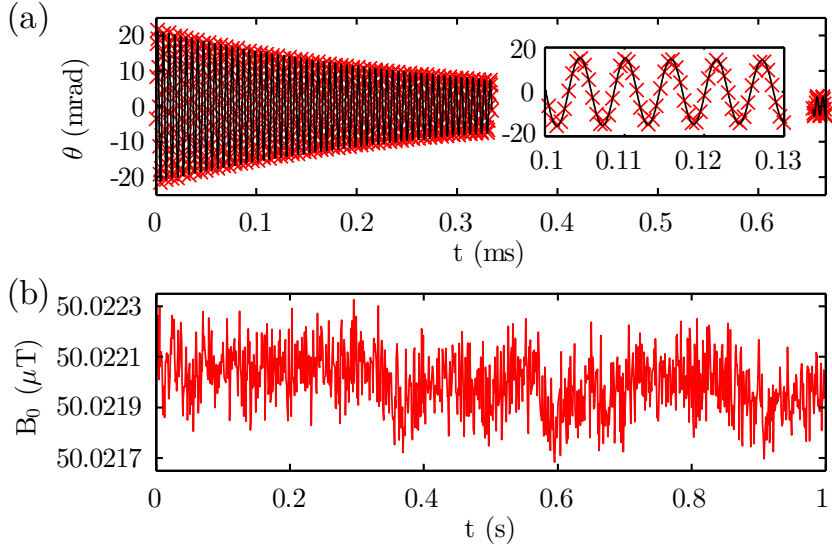

(c)

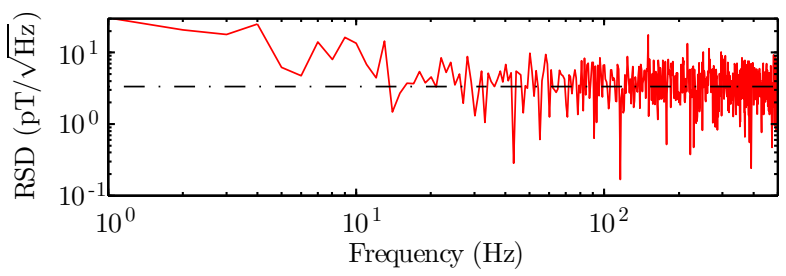

FIG. 7. (a) Typical FID signal (red) with corresponding model fit (black line) acquired using the AM configuration in a $50 \mu \mathrm{T}$ magnetic field. The inset shows $30 \mu \mathrm{s}$ of data to make the oscillations and fit visible. (b) Magnetic field data recorded for a period of $1 \mathrm{~s}$ by fitting adjacent signals in an FID train. (c) Calculated root spectral density (RSD) of the time domain magnetic field data. The dotted line indicates the noise floor calculated to be $3 \mathrm{pT} / \sqrt{\mathrm{Hz}}$ with a bandwidth of $500 \mathrm{~Hz}$.

a signal train; this would in turn decrease the magnetometer bandwidth. The CRLB condition for an angular frequency extracted from a discrete damped sinusoid, in the limit of high sampling rate, can be calculated as 17 19],

$$
\sigma_{\omega_{L}}^{2} \geq \frac{24}{\left(A_{\theta} / \sigma_{\theta}\right)^{2} N T_{r}^{2}} C
$$

where $A_{\theta} / \sigma_{\theta}$ is the signal-to-noise ratio, $\mathrm{N}$ is the number of data points and $\mathrm{C}$ is a corrective factor that takes into account the signal decay given by,

$$
C=\frac{N^{3}}{12} \frac{\left(1-z^{2}\right)^{3}\left(1-z^{2 N}\right)}{z^{2}\left(1-z^{2 N}\right)^{2}-N^{2} z^{2 N}\left(1-z^{2}\right)^{2}},
$$

where $z=e^{-\gamma_{2} / f s}$. It can be shown that the optimum measurement window occurs at $T_{r} \simeq 2 / \gamma_{2}$ therefore the minimized CRLB for extraction of the magnetic field is,

$$
\sigma_{B_{0}}^{2} \geq \frac{6 \gamma_{2}^{2}}{\gamma^{2}\left(A_{\theta} / \sigma_{\theta}\right)^{2} N} C
$$

The noise density $\rho_{B_{0}}$ is usually considered as a more meaningful determination of the magnetometer performance as it includes the bandwidth of the measurement. Assuming the noise is white then,

$$
\rho_{B_{0}}=\frac{\sigma_{B_{0}}}{\sqrt{f_{b w}}}=\sigma_{B_{0}} \sqrt{2 T}
$$

where $f_{b w}$ is the magnetometer bandwidth and $T$ is the total measurement time 16. The FID signal shown in Fig. 7 (a) was used to calculate the CRLB condition which was estimated to be $\rho_{B_{0}} \geq 2 \mathrm{pT} / \sqrt{\mathrm{Hz}}$ for a measurement time $T=1 \mathrm{~ms}$. A representation of the sensitivity in the frequency domain can also be delivered using the RSD of the magnetic signal as provided in Fig. 7 (c) showing a noise floor of $3 \mathrm{pT} / \sqrt{\mathrm{Hz}}$ which closely resembles the CRLB estimation.

\section{FREQUENCY MODULATION SCHEME}

Aside from the microfabricated vapor cell, the AM configuration contains numerous components that are incompatible with a miniaturized design. The primary motivation for conversion to a FM implementation was to minimize the number of components necessary to characterize and operate the magnetometer whilst maintaining a high level of performance and chip-scale compatibility. The FM system was developed using components that could be easily integrated into a packaged device. The key alteration is the application of a VCSEL as the solitary laser source; it is housed in a TO46 can however, these devices can be utilized in a compact die format comprising a $250 \mu \mathrm{m}$ square with a thickness of $150 \mu \mathrm{m}$. Their single mode output has a sufficiently narrow spectral linewidth as to resolve the $D_{1}$ line hyperfine structure. The VCSEL is placed inside an adjustable collimation mount with an aspheric lens to condition the divergent beam; although this is not essential in a compact system, it will improve performance especially when implementing a polarimeter as any light leakage is minimized and the same beam width falls on both photodiodes. The beam size also becomes particularly important when attempting to balance between efficient optical pumping and maintaining a large interaction volume especially in situations where the optical power is limited as is the case here.

The temperature and injection current are the two adjustable parameters that modify the output wavelength of the VCSEL. The temperature is typically set to $80^{\circ} \mathrm{C}$ to localise the $D_{1}$ transition at an injection current of $2 \mathrm{~mA}$; the quoted temperature stability of $0.06 \mathrm{~nm} /{ }^{\circ} \mathrm{C}$ was in good agreement with experimental measurements. Heating of the laser module is performed by a Peltier thermo-electric cooler (TEC) in conjuction with the Thorlabs TED200C PID temperature controller. A temperature sensor is placed in close proximity to the collimation mount allowing measurement of the VCSEL temperature. The bulk of material used in the heating process increases the thermal time constant enabling an extremely stable temperature which would not be the case in a chip-scale device. In this case, a more stringent PID control would be required or potentially part of the measurement process dedicated to locking onto the absorption line. The whole laser assembly is contained within an aluminium enclosure to restrict ambient temperature fluctuations and reduce EMI. The Thorlabs VLDC002 VCSEL driver is utilized as a current source 
(a)
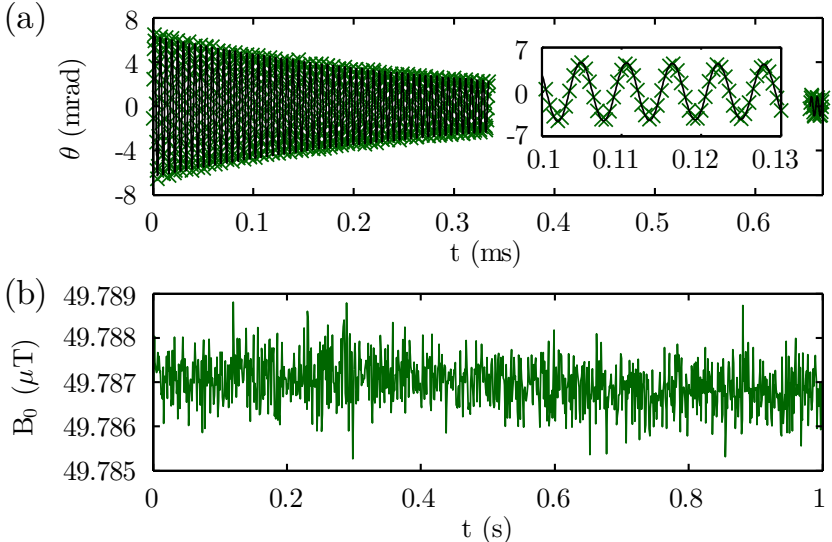

(c)

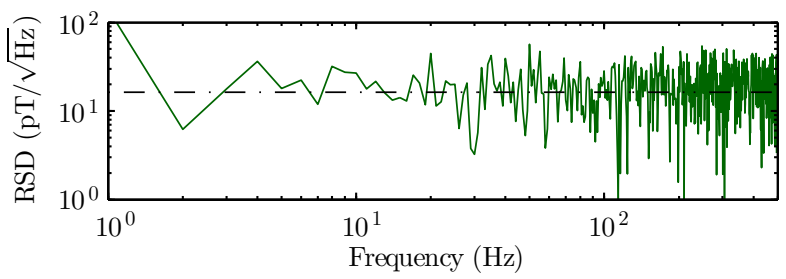

FIG. 8. (a) Typical FID signal (green) and corresponding fit (black line) acquired using the FM implementation; the experimental parameters external to the modulation were kept consistent with results from the AM scheme. (b) Magnetic field data acquired over a period of $1 \mathrm{~s}$ by fitting subsequent FID signals. (c) Calculating the RSD provides a noise floor of $16 \mathrm{pT} / \sqrt{\mathrm{Hz}}$ with a bandwidth of $500 \mathrm{~Hz}$.

allowing external voltage control of the output current as well as providing damage protection including a current limit. An arbitrary function generator acts as the voltage source enabling synchronous or single-pulse optical pumping such as that used in the AM scheme. The maximum injection current for single mode operation was implemented to improve the optical pumping efficiency.

The CRLB for the FM approach was calculated to be $\rho_{B_{0}} \geq 9 \mathrm{pT} / \sqrt{\mathrm{Hz}}$ for a measurement time $T=1 \mathrm{~ms}$ based on the fitted data shown in Fig. 8. The noise floor of the RSD, gained through analysis of the full signal train, estimated a sensitivity of $16 \mathrm{pT} / \sqrt{\mathrm{Hz}}$. The difference in sensitivity between the two systems is primarily a consequence of less efficient optical pumping in the FM regime as the maximum light power available from the VCSEL is an order of magnitude less than that of a diode laser. The lower level of spin coherence generated is easily observed in Figs. 7 (a) and 8 (a) by comparing the signal amplitude gained with both techniques.

\section{CONCLUSION AND OUTLOOK}

In summary, we have discussed the process involved in fabricating the sensor head consisting of a miniaturized Cs vapor cell with $\mathrm{N}_{2}$ buffer gas. This was utilized for magnetometry measurements in a FID setting using both the AM and FM techniques to monitor the free Larmor precession of the atomic spins. It was discovered that actively driving this precession using synchronous modula- tion provided consistent results even at higher bias fields where single-pulse optical pumping becomes increasingly inefficient. We used a balanced polarimeter to observe optical rotation, as opposed to traditional absorptionbased detection methods, resulting in sizeable noise reduction attributed to the differential measurement. Finally, we presented a comparison of the sensitivity performance for both modulation techniques; calculation of the CRLB yielded a noise density of $\rho_{B_{0}} \geq 2 \mathrm{pT} / \sqrt{\mathrm{Hz}}$ using the AM scheme and $\rho_{B_{0}} \geq 9 \mathrm{pT} / \sqrt{\mathrm{Hz}}$ for the FM implementation, whereas the noise floor of the RSD was measured to be $3 \mathrm{pT} / \sqrt{\mathrm{Hz}}$ and $16 \mathrm{pT} / \sqrt{\mathrm{Hz}}$ for the $\mathrm{AM}$ and FM configurations respectively. The less efficient optical pumping in the FM regime translates into a smaller SNR which directly impacts the sensitivity as described in Eq. (6).

A full optimization of the FM technique is still required which will include an extensive characterization of the ideal spectroscopic properties of the sensor head; the amount of $\mathrm{N}_{2}$ buffer gas content, vapor cell dimensions and operating temperature will be essential in enhancing the sensitivity by reducing the spin relaxation rate and maintaining a high SNR. There are additional opportunities for enhancement in how the magnetometer is driven for example the input polarization, beam width and frequency detuning could all be further optimized. Additionally, the gain characteristics of the transimpedance amplifiers in the balanced polarimeter should be tailored to the optical power output of the VCSEL which could provide improvements in SNR. VCSEL technology is still a field of considerable interest giving rise to constant improvements in the maximum output power possible. This is another potential source of improvement in the sensitivity of the FM system when operated in the lightnarrowed regime, resulting in perfomance that could rival that of the AM configuration but with a significantly higher degree of scalibility. Sinusoidal modulation was used as preliminary experiments displayed a larger SNR however, it was shown in Ref. [8] that with the correct modulation parameters a square waveform could increase the optical pumping efficiency.

A potential packaged device will contain a limited number of components including: a VCSEL, focussing lens, quarter-wave plate, Cs cell, PBS and two photodiodes. Thicker Cs cells will increase the spin coherence time and reduce the required cell temperature; this will improve the sensitivity performance and potentially power consumption. PBSs smaller than $2 \times 2 \times 2 \mathrm{~mm}^{3}$ are possible which is ideal for the beam sizes typically considered here if a focussing lens is applied. The photodiodes will likely be attached directly to each channel of the PBS and would be of similar dimensions. The smallest component is the VCSEL in a die format; machine placement of these devices is difficult especially as a specific orientation, with respect to the fast axis of the quarter-wave plate, is required to adequately balance the polarimeter detection system. Heater tracks can be placed directly on the Cs cells with a particular pattern that eradicates spurious magnetic fields from applied currents. RF heat- 
ing with a frequency considerably above the Larmor precession frequency and off-resonant laser heating are also possible alternatives. A straightforward control system could be implemented on FPGA for data acquisition, real-time processing, and to control the modulation input to the VCSEL; a feedback loop and sweep will maximize the FID amplitude in the synchronous regime. Singlepulse optical pumping will provide an estimate of the required modulation frequency and can also be used as the primary modulation technique at low bias fields. If the experimental parameters are fully optimized, a cmscale packaged device with pT level sensitivities should be possible in the FM implementation.

\section{ACKNOWLEDGEMENTS}

D. Hunter and S. Piccolomo acknowledge project funding from EPSRC through the CDT in applied photonics programme. We also acknowledge Texas Instruments for their funding and involvement in both projects. D. Hunter would like to thank Dr. Stuart Ingleby and Dr. Paul Griffin for many valuable discussions.
[1] W. E. Bell and A. L. Bloom, "Optical detection of magnetic resonance in alkali metal vapor," Phys. Rev. 107, 1559 (1957).

[2] W. E. Bell and A. L. Bloom, "Optically driven spin precession," Phys. Rev. Lett. 6, 280 (1961).

[3] D. Budker and M. Romalis, "Optical magnetometry," Nature Phys. 3, 227-234 (2007).

[4] W. F. Stuart, "Earth's field magnetometry," Rep. Prog. Phys. 35, 803 (1972).

[5] S. Groeger, G. Bison, P. E. Knowles, R. Wynands, and A. Weis, "Laser-pumped cesium magnetometers for highresolution medical and fundamental research," Sens. Actuator A-Phys. 129, 1-5 (2006).

[6] H. Xia, A. Ben-Amar Baranga, D. Hoffman, and M. V. Romalis, "Magnetoencephalography with an atomic magnetometer," Appl. Phys. Lett. 89, 211104 (2006).

[7] S. Knappe, P. D. D. Schwindt, V. Gerginov, V. Shah, L. Liew, J. Moreland, H. G. Robinson, L. Hollberg, and J. Kitching, "Microfabricated atomic clocks and magnetometers," J. Opt. A: Pure Appl. Opt. 8, S318 (2006).

[8] R. Jiménez-Martínez, W. C. Griffith, Y-J. Wang, S. Knappe, J. Kitching, K. Smith, and M. D. Prouty, "Sensitivity comparison of Mx and frequency-modulated Bell-Bloom Cs magnetometers in a microfabricated cell," IEEE Trans. Instrum. Meas. 59, 372-378 (2010).

[9] C. N. Johnson, P. D. D. Schwindt, and M. Weisend, "Multi-sensor magnetoencephalography with atomic magnetometers," Physics in Medicine \& Biology 58, 6065 (2013).

[10] J. Kitching, S. Knappe, P. D. D. Schwindt, V. Shah, L. Hollberg, L-A. Liew, and J. Moreland, "Power dissipation in a vertically integrated chip-scale atomic clock," in Frequency Control Symposium and Exposition, 2004. Proceedings of the 2004 IEEE International (IEEE, 2004) pp. 781-784.

[11] H. B. Dang, A. C. Maloof, and M. V. Romalis, "Ultrahigh sensitivity magnetic field and magnetization measurements with an atomic magnetometer," Applied Physics Letters 97, 151110 (2010).

[12] V. Gerginov, S. Krzyzewski, and S. Knappe, "Pulsed operation of a miniature scalar optically pumped magnetometer," JOSA B 34, 1429-1434 (2017).

[13] S. J. Seltzer, Developments in alkali-metal atomic magnetometry (Princeton University, 2008).

[14] S. Groeger, G. Bison, J-L. Schenker, R. Wynands, and A. Weis, "A high-sensitivity laser-pumped Mx magnetometer," Eur. Phys. J. D 38, 239-247 (2006).
[15] G. Di Domenico, G. Bison, S. Groeger, P. Knowles, A. S. Pazgalev, M. Rebetez, H. Saudan, and A. Weis, "Experimental study of laser-detected magnetic resonance based on atomic alignment," Phys. Rev. A 74, 063415 (2006).

[16] Z. D. Grujić, P. A. Koss, G. Bison, and A. Weis, "A sensitive and accurate atomic magnetometer based on free spin precession," Eur. Phys. J. D 69, 135 (2015).

[17] A. Moschitta and P. Carbone, "Cramér-rao lower bound for parametric estimation of quantized sinewaves," IEEE Trans. Instrum. Meas. 56, 975-982 (2007).

[18] Y-X. Yao and S. M. Pandit, "Cramér-Rao lower bounds for a damped sinusoidal process," IEEE Trans. Signal Process. 43, 878-885 (1995).

[19] C. Gemmel, W. Heil, S. Karpuk, K. Lenz, Ch. Ludwig, Yu. Sobolev, K. Tullney, M. Burghoff, W. Kilian, S. Knappe-Grüneberg, et al., "Ultra-sensitive magnetometry based on free precession of nuclear spins," Eur. Phys. J. D 57, 303-320 (2010).

[20] T. E. Dyer and N. L. Brockie, "Vapor cell and method for making same," (2017), US Patent 9,639,062.

[21] L-A. Liew, J. Moreland, and V. Gerginov, "Waferlevel filling of microfabricated atomic vapor cells based on thin-film deposition and photolysis of cesium azide," Appl. Phys. Lett. 90, 114106 (2007).

[22] S. Woetzel, V. Schultze, R. IJsselsteijn, T. Schulz, S. Anders, R. Stolz, and H-G. Meyer, "Microfabricated atomic vapor cell arrays for magnetic field measurements," Rev. Sci. Instrum. 82, 033111 (2011).

[23] P. Siddons, C. S. Adams, C. Ge, and I. G. Hughes, "Absolute absorption on rubidium D lines: comparison between theory and experiment," J. Phys. B: At. Mol. Opt. Phys. 41, 155004 (2008).

[24] D. A. Steck, "Cesium D line data," available online at http://steck.us/alkalidata (2010).

[25] G. A. Pitz, C. D. Fox, and G. P. Perram, "Pressure broadening and shift of the cesium D2 transition by the noble gases and $\mathrm{N}_{2}, \mathrm{H}_{2}, \mathrm{HD}, \mathrm{D}_{2}, \mathrm{CH}_{4}, \mathrm{C}_{2} \mathrm{H}_{6}, \mathrm{CF}_{4}$, and ${ }^{3}$ He with comparison to the D1 transition," Phys. Rev. A 82, 042502 (2010).

[26] G. A. Pitz, D. E. Wertepny, and G. P. Perram, "Pressure broadening and shift of the cesium D1 transition by the noble gases and $\mathrm{N}_{2}, \mathrm{H}_{2}, \mathrm{HD}, \mathrm{D}_{2}, \mathrm{CH}_{4}, \mathrm{C}_{2} \mathrm{H}_{6}, \mathrm{CF}_{4}$, and ${ }^{3}$ He," Phys. Rev. A 80, 062718 (2009).

[27] O. Kozlova, S. Guérandel, and E. de Clercq, "Temperature and pressure shift of the Cs clock transition in the presence of buffer gases: Ne, $\mathrm{N}_{2}$, Ar," Phys. Rev. A 83, 062714 (2011). 
[28] Y. Liu, J. Lin, G. Huang, Y. Guo, and C. Duan, "Simple empirical analytical approximation to the voigt profile," J. Opt. Soc. Am. B 18, 666-672 (2001).

[29] T. Scholtes, S. Woetzel, R. IJsselsteijn, V. Schultze, and H-G. Meyer, "Intrinsic relaxation rates of polarized cs vapor in miniaturized cells," Appl. Phys. B 117, 211218 (2014).

[30] H. G. Dehmelt, "Modulation of a light beam by precessing absorbing atoms," Phys. Rev. 105, 1924 (1957).

[31] W. Happer, "Optical pumping," Rev. Mod. Phys. 44, 169 (1972).

[32] R. Jiménez-Martínez, W. C. Griffith, S. Knappe, J. Kitching, and M. Prouty, "High-bandwidth opti- cal magnetometer," J. Opt. Soc. Am. B 29, 3398-3403 (2012).

[33] T. Scholtes, V. Schultze, R. IJsselsteijn, S. Woetzel, and H-G. Meyer, "Light-narrowed optically pumped Mx magnetometer with a miniaturized Cs cell," Phys. Rev. A 84, 043416 (2011).

[34] V. Shah and M. V. Romalis, "Spin-exchange relaxationfree magnetometry using elliptically polarized light," Phys. Rev. A 80, 013416 (2009).

[35] T. Scholtes, S. Pustelny, S. Fritzsche, V. Schultze, R. Stolz, and H-G. Meyer, "Suppression of spin-exchange relaxation in tilted magnetic fields within the geophysical range," Phys. Rev. A 94, 013403 (2016). 